\title{
Leucémie aiguë myéloïde : quand diagnostic clinique rime avec piège diagnostique. A propos de deux observations
}

\author{
Lafon A, Ahossi V, Larras P, Souquet F, Perrin D \\ Service d'Odontologie, CHU, Dijon, France \\ arnaud.lafon@chu-dijon.fr
}

La leucémie aigue myéloïde (LAM) constitue un envahissement médullaire par une prolifération diffuse de cellules hématopoïétiques malignes. Le caractère aigu est défini par l'apparition rapide, en quelques semaines, d'un tableau clinico-biologique d'insuffisance médullaire avec parfois, de rares manifestations buccales (Orazi 2007). Les auteurs se proposent, au travers de deux observations cliniques, de montrer qu'une LAM peut d'un côté se présenter de manière évidente, induisant d'emblée une confirmation biologique et, de l'autre, se présenter par des manifestations cliniques masquées par un contexte général n'évoquant pas d'emblée une LAM.

La première observation concerne une femme de 39 ans, adressée dans le Service d'Odontologie du CHU de Dijon par son chirurgien dentiste traitant, pour la prise en charge d'une hyperplasie gingivale généralisée. L'anamnèse relève une numération normale datant de deux mois, prescrite par son médecin traitant qui avait été consulté pour une tuméfaction gingivale. L'examen clinique met en évidence une altération de son état général avec hyperthermie, des adénopathies cervicales ainsi qu'une importante hyperplasie gingivale maxillaire et mandibulaire. Devant ce tableau, la patiente est hospitalisée. La numération de formule sanguine associée à un myélogramme révèle une LAM accompagnée d'une coagulation intravasculaire disséminée.

La deuxième observation concerne une femme enceinte, également âgée de 39 ans, en bon état général, sans antécédents médicaux. L'anamnèse note une succession récente de consultations spécialisées en gynécologie, en médecine générale à deux reprises, en chirurgie dentaire de ville et dans le Service d'Odontologie de Dijon. Toutes ses consultations ne relèvent aucune particularité, à part, pour certaines, une gingivite gravidique. Elle est adressée dans le Service d'Odontologie par le Service d'accueil des urgences, pour des algies buccales exacerbées, soulagées par la morphine. La patiente arrive dans le Service dans un état de somnolence mais consciente ; l'examen clinique montre une gencive marginale hémorragique et ulcéro-nécrotique par endroits. La patiente est hospitalisée pour réaliser un bilan, mais une aggravation brutale et soudaine de son état général accompagné de signes de souffrance neurologique, fait réaliser un CT-scan cérébral mettant en évidence une hémorragie entrainant quelques heures plus tard le décès de la patiente. Les résultats des examens biologiques montreront l'existence d'une LAM.

L'intérêt de cette communication est de montrer qu'à partir de signes cliniques très différents, tantôt exubérants tantôt discrets, le diagnostic reste le même alors que le pronostic est diamétralement opposé. Il ressort de cette expérience que toute expression buccale d'une pathologie générale doit être systématiquement bilantée dès sa première prise en charge médicale notamment odontologique (Van der Haring et al. 2006). 